\title{
Ultrasound guided fine needle aspiration cytology diagnosis of collision tumor in a lymph node: Lymphoma and metastatic adenocarcinoma
}

Qian Wang, Dhanalaksmi Balakrishnan, Maoxin Wu*

Department of Pathology, Stony Brook Medicine at Stony Brook University, Stony Brook, NY, United States

Received: January 20, 2017

DOI: $10.5430 /$ crcp.v4n2p1
Accepted: February 19, $2017 \quad$ Online Published: March 28, 2017

URL: https://doi.org/10.5430/crcp.v4n2p1

\begin{abstract}
Diagnosis of two distinct malignancies in a single sample is rare. Here we report a case of lung adenocarcinoma metastasized to a supraclavicular lymph node which was concomitantly diagnosed with chronic lymphocytic leukemia/small lymphocytic lymphoma (CLL/SLL) using ultrasound-guided fine needle aspiration biopsy (US-FNA) sample.

A 62-year-old Caucasian male presented with cough and bloody sputum. US-FNA of a supraclavicular lymph node showed two distinct cell populations and, based on morphological examination and immunohistochemical staining of the FNA cell block slides, diagnosed with CLL/SLL and metastatic lung adenocarcinoma. Flow cytometric analysis of peripheral blood supported the diagnosis of CLL/SLL. To our knowledge, this is a rarely reported cytology case in which metastatic lung adenocarcinoma is concurrently diagnosed with CLL/SLL in a single lymph node and can be diagnosed based an FNA sample.
\end{abstract}

Key Words: Leukemia, Lymphocytic, Chronic, B-cell, Adenocarcinoma, Collision tumor

\section{INTRODUCTION}

Synchronous neoplasms are unusual but not rare. However, the co-existence of more than one different type of malignancy at the same site is far less common, with even fewer reports about both malignancies diagnosed at the same time and same location. ${ }^{[1-8]}$ Herein we report a case where a lung adenocarcinoma metastasized to a supraclavicular lymph node was concomitantly diagnosed with chronic lymphocytic leukemia/small lymphocytic lymphoma (CLL/SLL) on the same ultrasound-guided fine needle aspiration biopsy (US-FNA) sample. To the best of our knowledge, this is the first cytology case report describing both metastatic lung adenocarcinoma and CLL/SLL diagnosed in a single aspirate sample.

\section{Case PResentation}

A 62-year-old male patient, current smoker, with no known past medical history, presented to our emergency department complaining of cough for one month with bloody sputum. He denied any fever, chills or shortness of breath. The initial chest CT scan revealed bilateral lymphadenopathy, consolidation of the left apical posterior segment and filling defect of a segmental branch of the left upper lobe. The major differential diagnoses included a mass or pulmonary embolism. At presentation, the patient was found to have leukocytosis $\left(45.33 \times 10^{6} / \mathrm{L}\right)$ with smudgy cells on the peripheral blood

\footnotetext{
*Correspondence: Maoxin Wu; Email: Maoxin.wu@stonybrookmedicine.edu; Address: Department of Pathology, Stony Brook Medicine at Stony Brook University, Stony Brook, NY 11794, United States.
} 
smear. He was admitted to medicine for further evaluation and management.

The left supraclavicular area was evaluated with a GE LOGIQe ultrasound machine and revealed an enlarged mixed echoic node in between two major blood vessels (see Figure 1). The node was measured to be $1.89 \mathrm{~cm} \times 1.12 \mathrm{~cm}$ on a transverse image. US-FNA of the node was performed and scant pink tan material was collected on one pass using a 27-gauge needle. An air dried Diff-Quick stained smear and an alcohol-fixed Papanicolaou-stained smear were prepared. Additional tissue/cells were saved in $10 \mathrm{ml}$ RPMI 1,640 medium for cell block preparation.

Cytomorphologic analysis (see Figure 2A and 2B) showed a cellular background of minimally atypical small lymphocytes with uniformly round, condensed nuclei and scant cytoplasm. Interspersed with this lymphocyte population were variably sized larger cell clusters. The larger cell nuclei demonstrated moderate to severe atypia and measured three to five times the size of normal red blood cells. H\&E stained cell block sections showed that these larger cells were pleomorphic with gland-forming architecture, thus favoring metastatic adenocarcinoma (see Figure 2C). Microscopic examination of the smears and cell block sections showed very rare neutrophils, while the patient's peripheral blood on the same day of the FNA procedure had $66 \%$ lymphocytes and $25 \%$ neutrophils. This indicated that the sample was collected from the node with insignificant blood contamination.

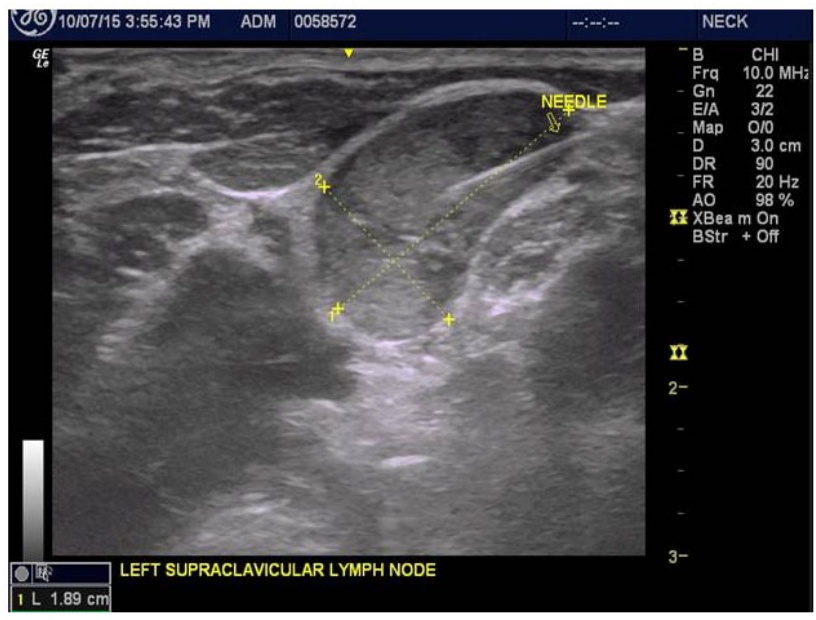

Figure 1. US-FNA biopsy of the left supraclavicular mass

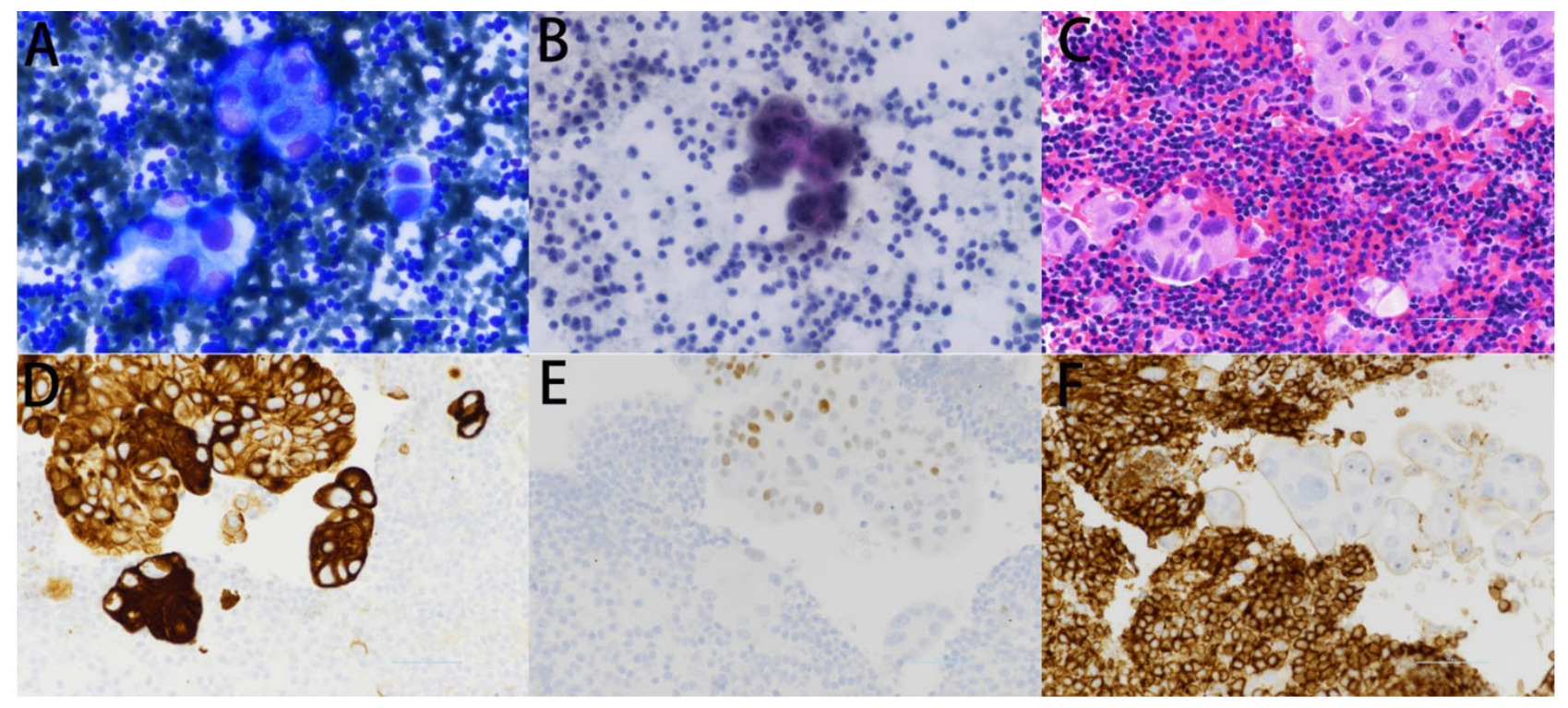

Figure 2. Representative cytological preparations and immunohistochemistry stains

(A, B) FNA smears showing small clusters of malignant cells present in a background of a monomorphic population of small atypical lymphocytes (A, Diff-Quick, 400×; B, Papanicolaou, 400×). (C) Cell block preparation demonstrating clusters of malignant cells consistent with adenocarcinoma surrounded by a dense monomorphic population of small atypical lymphocytes $(H \& E, 400 \times) .(D, E, F)$ Immunohistochemistry stains on sections of cell block revealing that the large malignant cells are positive for $C K 7(D, 400 \times)$ and TTF-1 (E, 400×); and the small atypical lymphoid cells positive for CD20 (F, 400×).

Immunocytochemistry (IHC) on cell block sections showed that the larger cells were positive for CK7 (see Figure 2D), TTF-1 (see Figure 2E), Napsin A and cyclinD1; but were negative for $\mathrm{p} 63$ and rarely positive for CDX-2 and CK20. The small atypical lymphoid cells were positive for CD20 (see Figure 2F), CD5 and CD23, and were negative for $\mathrm{Cy}-$ 
clinD1. CD3 was positive in the in between reactive $\mathrm{T}$ cells. Correlation of these findings with the cytomorphology led to a final diagnosis of metastatic lung adenocarcinoma in a collision with chronic lymphocytic lymphoma/leukemia.

Flow cytometric analysis of peripheral blood showed a predominant population of small to intermediate sized monoclonal B-cell lymphocytes (65\%) to be positive for CD5, CD19, CD20, CD23 and FMC7, while negative for CD10. The cells expressed surface immunoglobulin of the kappa light chain phenotype. The result suggests atypical chronic lymphocytic lymphoma.

Sections of cell block, which were also sent for fluorescence in situ (FISH) testing to detect ALK rearrangement and pyrosequencing for EGFR mutation, were all negative.

On most recent follow-up, the patient was found to have a metastatic spread of the pulmonary adenocarcinoma to his brain and bones. He is currently status post chemotherapy and radiation therapy.

\section{Discussion}

CLL/SLL is the most common leukemia among adults in the western hemisphere. It often co-expresses CD5 and CD23, while FMC7 and CD79b are usually negative or weakly expressed in typical cases of CLL/SLL, although FMC7 can be positive in about $15 \%$ of CLL patients who also have atypical cell morphology with bright CD20 and SIgG expression. Our patient was positive for CD5, CD20 and CD23 by IHC. This immuno-profile is consistent with the diagnosis of CLL/SLL.

Patients with CLL/SLL have an increased risk of developing a second primary neoplasm. The occurrence of a lung neoplasm in CLL/SLL patients is about $1.9 \%$ according to a study conducted at Memorial Sloan-Kettering Cancer Center from 1977 to $1998 .{ }^{[9]}$ These secondary neoplasms are usually diagnosed months or years after CLL/SLL is diagnosed.
The pathogenic mechanism could be mutagenic effects of radiation and chemotherapy, as well as the dysfunction of $\mathrm{B}$ cells. To our knowledge, there are only two cases reported about a concurrent lung neoplasm and CLL/SLL diagnosed at the same time. Both cases consist of squamous cell carcinoma of the lung. ${ }^{[2,4]}$ There is also a case of coincident adenocarcinoma and CLL/SLL in the lung mentioned in a retrospective study of 29 cytology cases, ${ }^{[10]}$ in which the collision tumor was shown on touch preparation from a concurrent corresponding surgical excision of a lung mass.

As a rapid, safe and relatively non-invasive procedure, FNA is an important diagnostic workup for many suspected neoplasms. With ultrasound guidance and on-site cytological evaluation, an FNA sample can be collected safely and be triaged appropriately for both cytomorphologic exam and ancillary studies. This case highlights the importance of being aware of the possibility of more than one neoplasm occurring in one site at the time of the procedure. This case proves to be essential to collecting sufficient material for ancillary studies such as immunohistochemistry for accurate diagnosis and molecular studies for guiding patient treatment. Flow cytometric analysis of the FNA sample might have provided additional support for diagnosing CLL/SLL. However, the authors focused more on saving sufficient material for immunohistochemical characterization and therapeutically relevant molecular markers of the metastatic carcinoma knowing that low-grade lymphoma can also be diagnosed based on immunohistochemical profiling.

\section{Conclusions}

In summary, cytopathologists who perform FNA procedures should be aware of the possibility of collision tumors. With clinical and radiological correlations, a cytopathologist should be able to provide accurate pathological diagnoses of such a tumor with a US-FNA biopsy sample.

\section{REFERENCES}

[1] McElroy C, Velilla R, Chaudhary H, et al. Fine-needle aspiration diagnosis of squamous cell carcinoma in a lymph node involved with small lymphocytic lymphoma: case report and review of the literature. Diagnostic Cytopathology. 2009; 37(1): 48-50. PMid: 18973126. https://doi.org/10.1002/dc. 20955

[2] Antic D, Dencic-Fekete M, Tomin D, et al. Monosomy 12 and deletion of 13q34 in a case of chronic lymphocytic leukemia with concomitant lung cancer. Vojnosanit Pregl. 2010; 67(10): 864-6. PMid: 21061845. https://doi.org/10.2298/VSP1010864A

[3] Gajendra S, Sharma R, Sahoo MK. Triple cancer: chronic lymphocytic leukemia with bladder and prostate carcinoma. Malays J Pathol. 2015; 37(2): 159-63. PMid: 26277675.
[4] Rovira Daudi E, Carrion Valero F, Garcia Diaz M, et al. Lung carcinoma and chronic lymphocytic leukemia: report of a synchronous diagnosis. Anales de medicina interna (Madrid, Spain: 1984). 1997; 14(1): 35-7. PMid: 9091034.

[5] Sur N, Silverman JF. Synchronous malignancies detected by effusion cytology. Diagnostic Cytopathology. 1998; 18(3): 184-7. PMid: 9523135. https ://doi.org/10.1002/(SICI) 1097-033 9 (199803) $18: 3<184::$ AID-DC4>3.0.CO ; 2-E

[6] Tse JY, Stover DG, Chebib I. Simultaneous metastasis of melanoma and lung adenocarcinoma to hilar lymph nodes and pleural effusion after renal transplantation. Cytopathology. 2014; 25(5): 342-4 http://dx.doi.org/10.1111/cyt.12088

[7] Shin HC, Gu MJ, Kim SW, et al. Coexistence of gastrointestinal 
stromal tumor and inflammatory myofibroblastic tumor of the stomach presenting as a collision tumor: first case report and literature review. Diagnostic Pathology. 2015; 10: 181. PMid: 26445324. http://dx.doi.org/10.1186/s13000-015-0413-y

[8] Caraway NP, Wojcik EM, Saboorian HM, et al. Concomitant lymphoma and metastatic carcinoma in a lymph node: diagnosis by fine-needle aspiration biopsy in two cases. Diagnostic Cytopathology. 1997; 17(4): 287-91. PMid: 9316785. https://doi.org/10.1002/(SICI) 1097-0339(199710)17: 4<287: : AID-DC9>3.0.C0;2-9
[9] Parekh K, Rusch V, Kris M. The clinical course of lung carcinoma in patients with chronic lymphocytic leukemia. Cancer. 1999; 86(9): 1720-3. PMid: 10547544. https://doi.org/10.1002/(SICI) 1 097-0142(19991101) 86: 9<1720: :AID-CNCR13>3.0.CO;2-X

[10] Kastenbaum HA, Khalbuss WE, Felgar RE, et al. The spectrum of coincident entities with small lymphocytic lymphoma/chronic lymphocytic leukemia (SLL/CLL) diagnosed by cytology. Cyto Journal. 2010; 7: 20. PMid: 20976208. https://doi.org/10.4103/1742 $-6413.70966$ 